\title{
Beware of density dependent pair potentials
}

\author{
A A Louis \\ Department of Chemistry, Lensfield Rd, Cambridge CB2 1EW, UK
}

Received 2 May 2002, in final form 8 July 2002

Published 27 September 2002

Online at stacks.iop.org/JPhysCM/14/9187

\begin{abstract}
Density (or state) dependent pair potentials arise naturally from coarse-graining procedures in many areas of condensed matter science. However, correctly using them to calculate physical properties of interest is subtle and cannot be uncoupled from the route by which they were derived. Furthermore, there is usually no unique way to coarse-grain to an effective pair potential. Even for simple systems like liquid argon, the pair potential that correctly reproduces the pair structure will not generate the right virial pressure. Ignoring these issues in naive applications of density dependent pair potentials can lead to an apparent dependence of thermodynamic properties on the ensemble within which they are calculated, as well as other inconsistencies. These concepts are illustrated by several pedagogical examples, including effective pair potentials for systems with many-body interactions, and the mapping of charged (Debye-Hückel) and uncharged (Asakura-Oosawa) two-component systems onto effective onecomponent ones. The differences between the problems of transferability and representability for effective potentials are also discussed.
\end{abstract}

\section{Introduction}

No known materials exist in nature whose behaviour can be completely captured by pair potentials alone. Even the properties of a noble gas like argon have a finite contribution from three-body Axilrod-Teller triple-dipole interactions [1]. Thus, the pair potentials used to describe condensed matter systems always arise from coarse-graining procedures, where a subset of the degrees of freedom of the full (quantum) statistical mechanical system are integrated out. In the aforementioned example, integrating the three-body interactions over angular coordinates results in effective parameters for the Lennard-Jones (LJ) pair potential, which will depend on state. Similarly, in metallic systems, integrating out the free electrons leads to a configuration independent volume term and pair potentials that depend on the global density [2]. Alternative coarse-graining procedures for metals such as the embedded atom method [3, 4], effective medium theory [5], Finnis-Sinclair potentials [6] or glue potentials [7] result in a local density or environment dependence.

Coarse-graining methods are also crucial to deriving tractable statistical mechanical treatments of soft-matter systems, where a large number of different length and timescales 
may coexist. An increasingly popular coarse-graining technique consists of deriving effective potentials and exploiting their analogy with well studied simple atomic or molecular systems to extract phase behaviour and correlations [8-10]. Again, these effective interactions are often reduced to an approximate pairwise description with parameters that depend on state.

Direct inversion from experimental structure factors are another way to derive the parameters for effective pairwise potentials $[11,12]$. These almost always show a dependence on state, especially for the case of soft-matter systems. This is not surprising, of course, since one can easily imagine that the interactions between two effective particles depend on the overall density. For example, changing the concentration of a micellar solution may affect the internal structure of the micelles, which in turn leads to a density dependence of the effective pair interaction between the particles.

That an effective pair potential derived in one context does not always perform well in another is well known, and usually categorized as a problem of transferability. For example, if the parameters of an effective pair potential depend on density, then a parametrization of the potential at $\rho_{1}$ is not the same as the one needed at a different density $\rho_{2}$ - the potential at $\rho_{1}$ is not transferable to the state point at $\rho_{2}$. Because it is usually hard to derive an explicit state dependence, a given potential is often used for state points close enough to the one for which it was parametrized that transferability problems are not deemed to be important. In this case the potential is usually treated as if it were independent of state.

What I will endeavour to show in the present paper is that there are deeper problems associated with the use of effective pair potentials, even when the problem of transferability appears to be solved. These include problems of representability: at a given state point, no single pair potential may exist that can capture all the properties of a given material. The particular example of state dependence studied is pair potentials that depend on the global density $\rho$ as $v(r ; \rho)^{1}$. The paper's focus is partially pedagogical. For that reason rather simple models are treated, with a special emphasis on the liquid phase. Some of these results have already appeared in one form or another in the literature, and will be briefly reviewed.

The paper is organized as follows: section 2 describes the apparent inconsistencies that arise between the virial and compressibility routes to thermodynamics for a simple density dependent pair potential $v(r ; \rho)$. Section 3 discusses the effective pair potentials that result from integrating out third and higher order many-body interactions. The effective pair potential that correctly describes the excess internal energy is shown to be different from the one that correctly describes the pair structure. These points are illustrated with a specific application from polymer solutions. In section 4 the McMillan-Mayer [13] tracing-out procedure is analysed for an exactly solvable lattice version of the Asakura-Oosawa (AO) [14] model. While this procedure maps onto a useful effective one-component picture in the semi-grand ensemble, integrating out the smaller particles in a canonical ensemble does not lead to an effective Hamiltonian decomposable as a sum over independent interactions. For charged systems the canonical ensemble is the natural choice to integrate out microscopic co- and counterions. Again, apparent ambiguities arise when the density dependent Debye-Hückel potential is used to derive thermodynamics. Finally, conclusions from these different model calculations are summarized in section 5.

\section{General thermodynamic inconsistencies from a naive application of density dependent pair potentials}

As a first rather general example, consider a homogeneous fluid in a volume $V$, whose $N$ particles interact with a spherically symmetric pair potential $v(r ; \rho)$, which depends on the

1 Effective potentials could also depend on other state variables like temperature $T$, but that will not be treated here. 
global density $\rho=N / V$. There are no volume, one-body or many-body terms. No further assumptions as to the origin of the density dependence are made. Two established ways to calculate the equation of state (EOS) $Z$ and other thermodynamic properties from the correlation functions are [15]:

(i) The compressibility route

$$
Z_{c}=\frac{\beta P}{\rho}=\int_{0}^{\rho} \frac{\partial \beta P\left(\rho^{\prime}\right)}{\partial \rho^{\prime}} \frac{\mathrm{d} \rho^{\prime}}{\rho}=\int_{0}^{\rho}\left[1-\rho^{\prime} \hat{c}\left(k=0 ; \rho^{\prime}\right)\right] \frac{\mathrm{d} \rho^{\prime}}{\rho}
$$

where $\hat{c}\left(k=0 ; \rho^{\prime}\right)$ is the zero-wavelength component of the Fourier transform (FT) of the direct correlation function $c(r), \beta=1 / k_{B} T$, and $P$ is the pressure. This relationship follows from simple properties of the correlation functions and their connections to thermodynamics in the grand canonical ensemble-it is therefore independent of the particular form of the interactions between the particles, which need not be pairwise additive [15].

(ii) The virial route

$$
Z_{v i r}^{\rho}=\frac{\beta P}{\rho}=1-\frac{2}{3} \beta \pi \rho \int_{0}^{\infty} r^{2}\left\{r \frac{\partial v(r ; \rho)}{\partial r}-3 \rho \frac{\partial v(r ; \rho)}{\partial \rho}\right\} g(r) \mathrm{d} r
$$

where $g(r)$ is the radial distribution function. The standard way to derive the virial equation is directly through the canonical partition function

$$
Q(N, V, T)=\frac{\Lambda^{-3 N}}{N !} \int \mathrm{d} \boldsymbol{r}^{N} \exp \left\{-\beta \sum_{i<j} v\left(r_{i j} ; \rho\right)\right\}
$$

where $\Lambda$ is the usual thermal de Broglie wavelength. The volume derivative in

$$
\beta P=\left(\frac{\partial \log Q(N, V, T)}{\partial V}\right)_{N, T}
$$

also acts directly on the pair potential, which brings in the extra $\partial v(r ; \rho) / \partial \rho$ term in the virial equation (2), a result first pointed out in 1969 by Ascarelli and Harrison [16] in the context of density dependent pair potentials used for modelling liquid metals. This particular form of the virial equation is only valid for pair potentials, but the derivation of generalizations for systems with three-body terms is straightforward.

So far so good: both the compressibility equation (which does not change from the density independent case) and the virial equation (which does) appear to be derived for the case of a density dependent pair potential. Nevertheless, this apparent rigour deceives, since it is trivial to find density dependent pair potentials where the two routes generate different thermodynamics. Consider, for example, a special class of density dependent pair potentials with

$$
v(r ; \rho)=\epsilon(\rho) v^{0}(r)
$$

Two possible $\epsilon(\rho)$ are shown in figure 1. The compressibility equation (1) results in a different $Z$ at $\rho=\rho^{*}$ for potentials (a) and (b), since the effects of all densities below $\rho^{*}$ are relevant. In contrast, the virial equation (2) cannot distinguish between the two potentials at $\rho=\rho^{*}$ because it only includes a local density dependence. Of course it is not surprising that the two routes to thermodynamics disagree, since one was derived in the canonical ensemble, which only samples a single global density, while the other was derived in the grand-canonical ensemble, which samples all densities. 


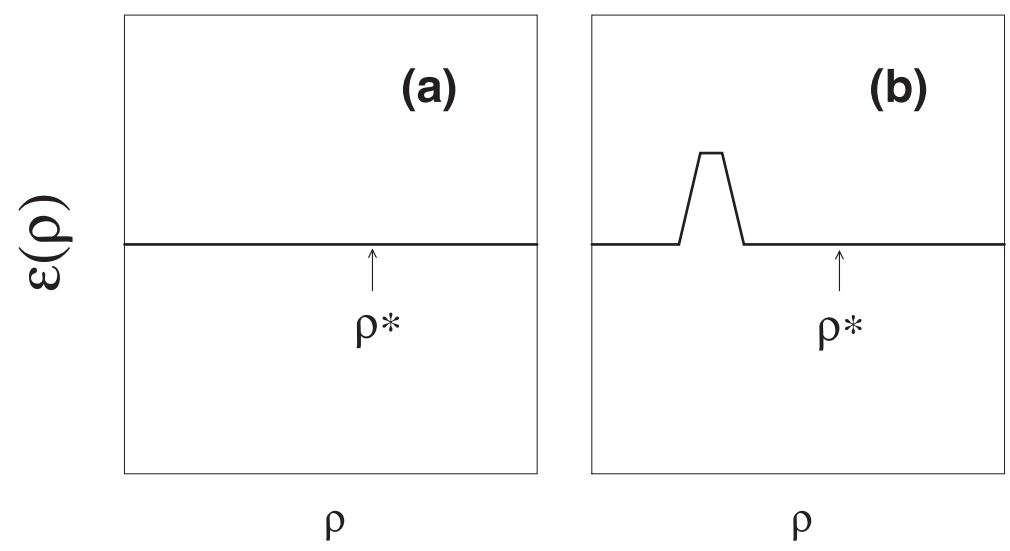

Figure 1. Amplitude $\epsilon(\rho)$ for the simple potential $v(r ; \rho)=\epsilon(\rho) v^{0}(r)$. Potentials (a) and (b) are equivalent except on a small density interval. While the compressibility route to the EOS can distinguish between the two potentials at $\rho^{*}$, the virial route cannot.

However, the origin of the discrepancy lies deeper than that. One might think that the virial equation is less reliable, since it only treats the density dependence as a local derivative. But, as the following example will show, the two routes disagree even for a simple linear density dependence; even worse, the density dependence correction of Ascarelli and Harrison [16] corrects in the wrong direction-it makes the discrepancy between the two routes worse.

Consider a fluid interacting via the simple Gaussian potential

$$
\begin{aligned}
& v(r ; \rho)=\epsilon(\rho) \exp \left[-r^{2}\right] \\
& \epsilon(\rho)=\epsilon_{0}+\epsilon_{1} \rho
\end{aligned}
$$

which could be viewed as a model for interactions between polymer coils $[17,18]$. For small $\epsilon(\rho)$, this potential falls into the class of mean-field fluids [10,19-21], for which the simple random phase approximation (RPA) $c(r ; \rho) \approx v(r ; \rho)$ is very accurate and even becomes asymptotically exact in the limit of small $\epsilon(\rho)$ or large $\rho$. For integrable density independent potentials $v(r)$, the compressibility route and the RPA closure lead to an EOS of the mean-field $\left(Z_{M F}\right)$ form

$$
Z_{c}^{R P A}=Z_{M F}=1+\frac{1}{2} \rho \beta \hat{v}(k=0)
$$

where $\hat{v}(k)$ is the FT of the potential. More generally, for the same integrable density independent potentials, the virial route (2) reduces to

$$
Z_{v i r}^{0}=Z^{M F}-\frac{2}{3} \beta \pi \rho \int_{0}^{\infty} r^{2}\left\{r \frac{\partial v(r ; \rho)}{\partial r}\right\} h(r) \mathrm{d} r
$$

where $h(r)=g(r)-1$. In the limit of small $\hat{v}(k=0)$ or high densities, the second, integral term tends to zero so that the two routes to $Z$ approach each other [19]; the RPA closure approximation is then nearly self-consistent.

For the density dependent $v(r ; \rho)$ given by equation (6), the compressibility equation (1) takes on a simple form:

$$
Z_{c}^{R P A}=1+\pi^{3 / 2}\left(\frac{1}{2} \epsilon_{0} \rho+\frac{1}{3} \epsilon_{1} \rho^{2}\right) .
$$

The virial route without density dependent corrections, i.e. equation (9), results in

$$
Z_{v i r}^{0} \approx Z_{M F}=1+\pi^{3 / 2}\left(\frac{1}{2} \epsilon_{0} \rho+\frac{1}{2} \epsilon_{1} \rho^{2}\right)
$$




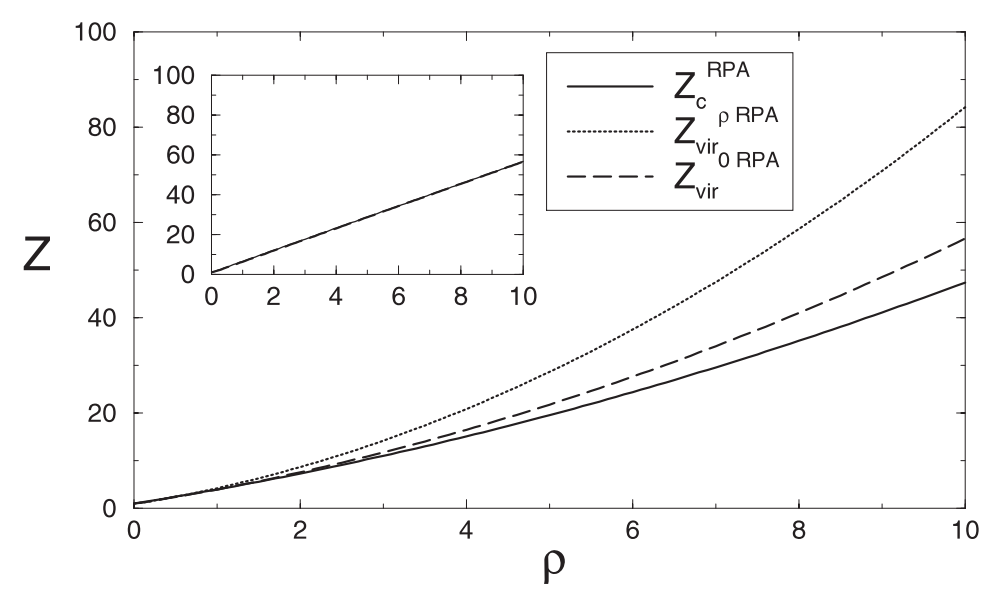

Figure 2. Comparison of three routes to thermodynamics for an effective potential $\beta v(r ; \rho)=$ $(1+0.1 \rho) \exp \left[-r^{2}\right] . \quad Z_{c}^{R P A}$ is given by equation $(10), Z_{v i r}^{\rho R P A}$ comes from an analytical RPA solution [22] to the virial equation (2) and $Z_{v i r}^{0 R P A}$ from an analytical RPA solution [19] to the simpler virial equation (9). The density dependent correction to the virial equation increases the disagreement between $Z_{c}$ and $Z_{v i r}$. Inset: in contrast to the density dependent case, the RPA virial and compressibility routes to $Z$ for $v(r)=2 \exp \left[-r^{2}\right]$ agree within the accuracy of the graph, demonstrating the near self-consistency of the closure for such potentials.

for the limit of small $\hat{v}(k=0)$ or large $\rho$. Within the RPA, the corrections to equation (11) from the second term in equation (9) can be analytically calculated for Gaussian potentials [19] and explicitly shown to be small for the limit being considered.

At this level, the two routes clearly do not agree. If $\epsilon_{1}>0$ then $Z_{v i r}^{0}>Z_{c}$, while if $\epsilon_{1}<0$ then $Z_{v i r}^{0}<Z_{c}$. One might argue that the discrepancy should stem from ignoring the density derivative term in the virial equation (2). But the opposite is true. Adding the density derivative correction results in

$$
Z_{v i r}^{\rho}=Z_{v i r}^{0}+2 \pi \rho^{2} \int r^{2} g(r) \epsilon_{1} \exp \left[-r^{2}\right] \mathrm{d} r .
$$

Since $g(r) \geqslant 0, \epsilon_{1}>0$ implies that $Z_{v i r}^{\rho}>Z_{v i r}^{0}>Z_{c}$, and $\epsilon_{1}<0$ implies that ${ }^{2} Z_{v i r}^{\rho}<Z_{v i r}^{0}<$ $Z_{c}$. In other words, the full virial equation (2), derived explicitly from the canonical ensemble with a density dependent potential, results in worse agreement than virial expressions (9) or (11) which ignore the density derivative terms. These points are illustrated in figure 2 for a particular example of the potential (6).

The problem clearly lies deeper than the fact that the virial equation only contains a local density derivative, since, for the example potential (6), this should be sufficient to describe all the density dependence. The next sections provide a partial answer to this apparent conundrum by explicitly deriving density dependent potentials, and showing that they should really be viewed as mathematical constructs whose physical interpretation cannot be separated from the way in which they were derived.

\section{Example 1. Effective pair potentials from many-body interactions}

This section demonstrates both in general and with an explicit example that there is no unique way to represent averages over many-body interactions as averages over a single pair potential

2 The conclusions for the relative ordering of $Z_{v i r}$ and $Z_{c}$ for $\epsilon_{1}>1$ hold more generally for all such potentials with $\partial \epsilon(\rho) / \partial \rho>1$ and vice versa. 
that depends on the global density $\rho$. Although this has already been pointed out several times in the literature [23-26], mainly in the context of noble gases, it is worth partially repeating because it often seems forgotten.

\subsection{Effective density dependent pair potentials from three-body potentials}

Consider a homogeneous one-component fluid system interacting with a density independent pair potential $w^{(2)}\left(\boldsymbol{r}_{i}, \boldsymbol{r}_{j}\right)$ and triplet potential $w^{(3)}\left(\boldsymbol{r}_{i}, \boldsymbol{r}_{j}, \boldsymbol{r}_{k}\right)$. The Hamiltonian can be written as

$$
H=K+\sum_{i<j} w^{(2)}\left(\boldsymbol{r}_{j}, \boldsymbol{r}_{j}\right)+\sum_{i<j<k} w^{(3)}\left(\boldsymbol{r}_{i}, \boldsymbol{r}_{j}, \boldsymbol{r}_{k}\right)
$$

with $K$ the kinetic energy operator. By using this Hamiltonian in a standard canonical partition function, the following exact expression can be derived for the excess internal energy $U$ :

$U(N, V, T)=\frac{1}{2} \rho^{2} \int \mathrm{d} \boldsymbol{r}_{1} \int \mathrm{d} \boldsymbol{r}_{2} g_{12} w_{12}+\frac{1}{6} \rho^{3} \int \mathrm{d} \boldsymbol{r}_{1} \int \mathrm{d} \boldsymbol{r}_{2} \int \mathrm{d} \boldsymbol{r}_{3} g_{123} w_{123}$

where $w_{12}=w^{(2)}\left(\boldsymbol{r}_{1}, \boldsymbol{r}_{2}\right), w_{123}=w^{(3)}\left(\boldsymbol{r}_{1}, \boldsymbol{r}_{2}, \boldsymbol{r}_{3}\right) . \quad g_{12}=g^{(2)}\left(r_{12}\right)$ and $g_{123}=$ $g^{(3)}\left(r_{12}, r_{13}, r_{23}\right)$ are the homogeneous pair and triplet radial distribution functions respectively, and $r_{i j}$ is the distance between particle $i$ and particle $j$

Three-body interactions are often cumbersome to use; an effective pair potential which reproduces the properties of the full system would be much more convenient. A popular method to achieve this consists of calculating the internal energy of the system governed by the original many-body Hamiltonian through an accurate method (like equation (14)), and then finding an effective pair potential $v_{U}^{e f f}\left(r_{i j} ; \rho\right)$ that reproduces the same energy at the same state point. For the system at hand, this can be done explicitly $[23,24,26]$ by writing equation (14) as

$$
U(N, V, T)=\frac{1}{2} \rho^{2} \int \mathrm{d} \boldsymbol{r}_{1} \int \mathrm{d} \boldsymbol{r}_{2} g_{12}\left(r_{12}\right) v_{U}^{e f f}\left(r_{12} ; \rho\right)
$$

which defines the effective pair potential

$$
v_{U}^{e f f}\left(r_{i j} ; \rho\right)=w_{12}\left(r_{i j}\right)+\delta v_{U}\left(r_{i j} ; \rho\right)
$$

where

$$
\delta v_{U}\left(r_{i j} ; \rho\right)=\frac{1}{3} \rho \int \mathrm{d} \boldsymbol{r}_{3} g_{13} g_{23} G_{123} w_{123}
$$

is the density dependent correction to the bare pair potential $w_{12}(r)$. Here $G_{123}$ is defined as the correction to the Kirkwood superposition approximation [27] for the triplet radial distribution function:

$$
g_{123}=g_{12} g_{13} g_{23} G_{123} \text {. }
$$

In contrast to the virial equation (2) of the previous section, re-deriving the two-body energy equation for a general $v(r ; \rho)$ within the standard canonical ensemble route [15] does not result in extra density dependent terms. In other words, within the canonical ensemble, an effective density dependent pair interaction can be constructed that correctly captures-by averages over pair correlations alone-the internal energy $U(N, V, T)$ of a system with twoand three-body interactions. Nevertheless, $v_{U}^{e f f}(r ; \rho)$ is not sufficient for a complete pairwise description. The three-body interaction $w_{123}$ also modifies $g_{12}$, and although $v_{U}^{e f f}(r ; \rho)$ generates a different $g_{12}$ than would be found by using only the bare pair potential $w_{12}(r)$, the pair correlations are not those of the original system, as will be shown below. 
A very useful theorem facilitating the study of pair correlations states that for a given homogeneous many-body system at a global density $\rho$, there exists a one to one mapping between a unique pair potential $v_{g}^{\text {eff }}(r ; \rho)$ and the $g^{(2)}(r)$ at that density [28] $]^{3}$. While the explicit analytical construction of $v_{g}^{e f f}(r ; \rho)$ is not as straightforward as that of its energy analogue $v_{U}^{\text {eff }}(r ; \rho)$, an expansion to lowest order in density and $w_{123}$ can be derived $[24,25,30,31]$ :

$v_{g}^{e f f}\left(r_{12} ; \rho\right) \approx w_{12}\left(r_{12}\right)+\delta v_{\rho}\left(r_{12} ; \rho\right)=w_{12}\left(r_{12}\right)+\rho \int \mathrm{d} r_{3}\left\{1-\exp \left(-w_{123}\right)\right\} g_{13} g_{23}$.

Comparing the density dependent corrections to $v_{g}^{\text {eff }}(r ; \rho)$ and $v_{U}^{\text {eff }}(r ; \rho)$ in the limit of small $\rho$ and weak $w_{123}$ leads to

$$
\frac{\delta v_{U}(r ; \rho)}{\delta v_{g}(r ; \rho)}=\frac{1}{3}+\mathcal{O}\left(w_{123}^{2} ; \rho^{2}\right) .
$$

To lowest order, the two density dependent corrections differ by a factor of three!

The unique one-to-one correspondence between $g^{(2)}(r)$ and $v_{g}^{\text {eff }}(r ; \rho)$ therefore implies that $v_{U}^{e f f}(r ; \rho)$ cannot reproduce the correct pair correlations for use in equation (15). This proves that the pair potential derived from the energy equation for a many-body system cannot completely and self-consistently capture the excess energy within a pairwise description; $v_{g}^{e f f}(r ; \rho)$ is also needed to generate the correct $g^{(2)}(r)$. On the other hand, because the compressibility equation (1) is independent of the underlying interactions, $v_{g}^{\text {eff }}(r ; \rho)$ is sufficient to derive the true compressibility, and from it other thermodynamic quantities of interest, within a purely pairwise description. But the price to pay for coarse-graining a manybody system to an effective two-body system in this way is that the thermodynamics can only be calculated along one specific route. Neither $v_{g}^{\text {eff }}(r ; \rho)$ nor $v_{U}^{\text {eff }}(r ; \rho)$ have a well defined physical meaning independent of the way in which they were derived. The arguments of this subsection are not new, as the following quote, made over 30 years ago in exactly the same context, demonstrates:

'We record our opinion that the use of density dependent effective pair potentials can be misleading unless it is recognized that these are mathematical constructs to be used in specified equations rather than physical quantities' [23].

\subsection{A worked example: polymers as soft colloids}

The concepts of the previous section can be made more concrete by examining a recent coarse-graining of polymers as soft colloids $[17,18,32]$. This system has the advantage that many-body correlations and interactions can be accurately calculated by computer simulation, allowing detailed comparisons of the coarse-grained system with the original many-body system.

The first step in the coarse-graining procedure is to choose an effective coordinate for the polymers, which we take to be the centre of mass (CM). The next step is to integrate out the monomeric degrees of freedom to derive an effective interaction between the polymer CMs. Following the discussion in [10,32], the Helmholtz free energy $\mathcal{F}$ of a set of $N$ polymers of length $L$ in a volume $V$, with their CMs distributed according to the set of coordinates $\left\{\boldsymbol{r}_{i}\right\}$, can be written as the following expansion:

3 Note that this pair potential cannot simultaneously represent the correct three-body correlations [29]. See e.g. [32] for a detailed example and discussion of this point. 


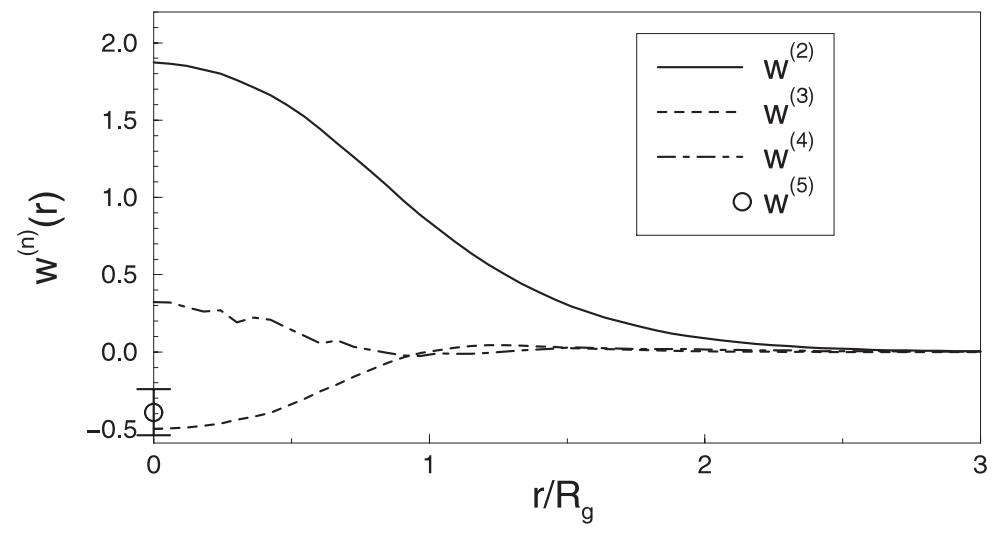

Figure 3. Effective potentials $w^{(n)}(r)$ between the CM of $L=500$ self-avoiding walk polymer coils. The coordinate $r$ denotes the pair distance for $n=2$, the length of an equilateral triangle for $n=3$ and the length of a tetrahedron for $n=4$. For $n=5$ only the interaction at full overlap was calculated (taken from [32]).

$$
\begin{aligned}
\mathcal{F}\left(N, V,\left\{\boldsymbol{r}_{i}\right\}\right) & =\mathcal{F}^{(0)}(N, V)+\sum_{i<j}^{N} w^{(2)}\left(\boldsymbol{r}_{i}, \boldsymbol{r}_{j},\right)+\sum_{i<j<k}^{N} w^{(3)}\left(\boldsymbol{r}_{i}, \boldsymbol{r}_{j}, \boldsymbol{r}_{k}\right) \\
& +\cdots+w^{(N)}\left(\boldsymbol{r}_{i}, \boldsymbol{r}_{j} \ldots \boldsymbol{r}_{N}\right) .
\end{aligned}
$$

In the scaling limit, each term in the series is independent of $L$ as long as the $n$-tuple CM coordinates $\left\{\boldsymbol{r}_{1}, \boldsymbol{r}_{2} \ldots \boldsymbol{r}_{n}\right\}$ are expressed in units of $R_{g}$, the radius of gyration at zero density. This coarse-grained free energy contains an implicit statistical average over all the monomeric degrees of freedom for a fixed set of CM coordinates $\left\{\boldsymbol{r}_{i}\right\} . \mathcal{F}^{(0)}(N, V)$ is the zero-body volume term, related to the internal free energy of a single polymer; translational symmetry implies that there is no explicit one-body term. The pair and higher body terms are defined in the standard way: the $n$th body term $w^{(n)}\left(\boldsymbol{r}_{1}, \boldsymbol{r}_{2} \ldots \boldsymbol{r}_{i_{n}}\right)$ for a particular set of $n$ CM coordinates is given by the total free energy $\mathcal{F}$ for $n$ polymers at those coordinates, minus the sum of all the lower order terms $[10,32]$. These interactions can be explicitly calculated by computer simulation. Examples are shown in figure 3, taken from [32], where further details can be found. The relative importance of each term decreases for increasing $n$, so that in principle the many-body expansion (21) is expected to converge [32].

The free energy $F(N, V)$ of the underlying polymer system follows from a final trace over all CM coordinates

$$
F(N, V)=-\ln \sum_{\left\{\boldsymbol{r}_{i}\right\}} \exp \left[-\mathcal{F}\left(N, V,\left\{\boldsymbol{r}_{i}\right\}\right)\right]
$$

so that equation (21) can be viewed as an expansion of the effective interaction between the CM in terms of (entropic) many-body interactions, in a close analogy to expansion of the energy of atomic or molecular systems in many-body interactions [33].

But in practice following this path is extremely cumbersome, because the number of coordinates and concomitant complexity of the interactions $w^{(n)}$ grows rapidly with increasing order $n$. To circumvent this problem one could simply truncate the expansion (21) at the pair level, but this would completely ignore the many-body interactions. Instead, we recently proposed $[17,18,32]$ a coarse-graining method which takes the many-body interactions into account in an average way. First, at a given $\rho$, the $g(r)$ between the CM of a polymer was 

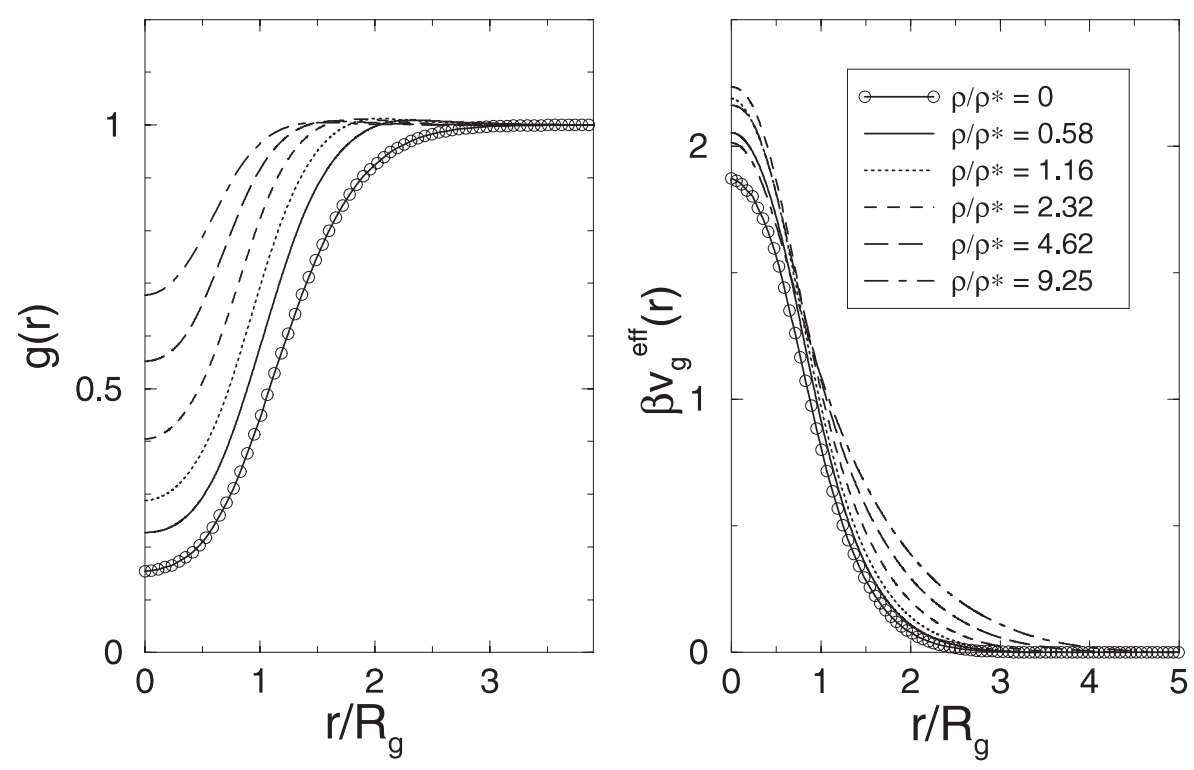

Figure 4. The effective polymer pair potentials $v_{g}^{\text {eff }}(r ; \rho)$, derived at different densities $\rho / \rho^{*}$ from an Ornstein-Zernike inversion of the CM pair distribution functions $g(r)$ of $L=500$ self-avoiding walk polymer coils (taken from [32]).

generated by computer simulations. Next, for each density, an Ornstein-Zernike integral equation approach was used to invert the $g(r)$ and generate the unique [28] effective pair potential $v_{g}^{\text {eff }}(r ; \rho)$ which exactly reproduces $g(r)$. Explicit examples of $v_{g}^{\text {eff }}(r ; \rho)$ are shown in figure 4 for different densities $\rho / \rho^{*}$, where $\rho^{*}=3 /\left(4 \pi R_{g}^{3}\right)$ denotes the crossover from the so-called 'dilute' to the 'semi-dilute' regimes [34]. For $\rho \rightarrow 0, v_{g}^{\text {eff }}(r ; \rho) \rightarrow w^{(2)}(r)$ while the difference $\delta v_{g}(r ; \rho)=v_{g}^{e f f}(r ; \rho)-w^{(2)}(r)$ grows with increasing density.

For the polymer system, the lowest order of the expansion (19) for the density dependent correction to the effective pair potential, $\delta v_{g}(r ; \rho)$, can be explicitly calculated [32]. The results, plotted in figure 5, show that despite the existence of higher order interactions $w^{(n)}$ with $n>3$, this weak $w^{(3)}$ and small $\rho$ form performs remarkably well, demonstrating that, for a polymer solution, the three-body interactions are the dominant cause of the density dependence in $v_{g}^{\text {eff }}(r ; \rho)$ at least for $\rho / \rho^{*} \leqslant 1$.

Since $v_{g}^{\text {eff }}(r ; \rho)$ was explicitly constructed to reproduce the correct pair correlations, it follows that the true thermodynamics of the full many-body system should be reproduced by using this potential in the compressibility equation (1). The results shown in figure 6 confirm this: full polymer simulations of the EOS $Z$ for $L=500$ and 2000 self-avoiding walk polymers on a cubic lattice compare well with the $\operatorname{EOS} Z_{c}$ calculated with the appropriate $v_{g}^{\text {eff }}(r ; \rho)$. The small residual differences at large $\rho$ are most likely due to numerical difficulties in performing accurate inversions at these high densities [35].

We now have a concrete example where the explicit construction of $v_{g}^{\text {eff }}(r ; \rho)$ leads to the full thermodynamics of a many-body system within a purely pairwise description. But what happens when this density dependent pair potential is used within the virial equation (2)? Figure 6 shows that the virial equation without the explicit density derivative is accurately approximated by the mean-field form $Z_{v i r}^{0} \approx Z_{M F}=1+\rho \hat{v}(k=0 ; \rho)$. Just as was found for positive $\epsilon_{1}$ in section $2, Z_{v i r}^{0}$ overestimates the true $Z$, and adding the density dependent correction (not shown) results in a significantly larger overestimate. 


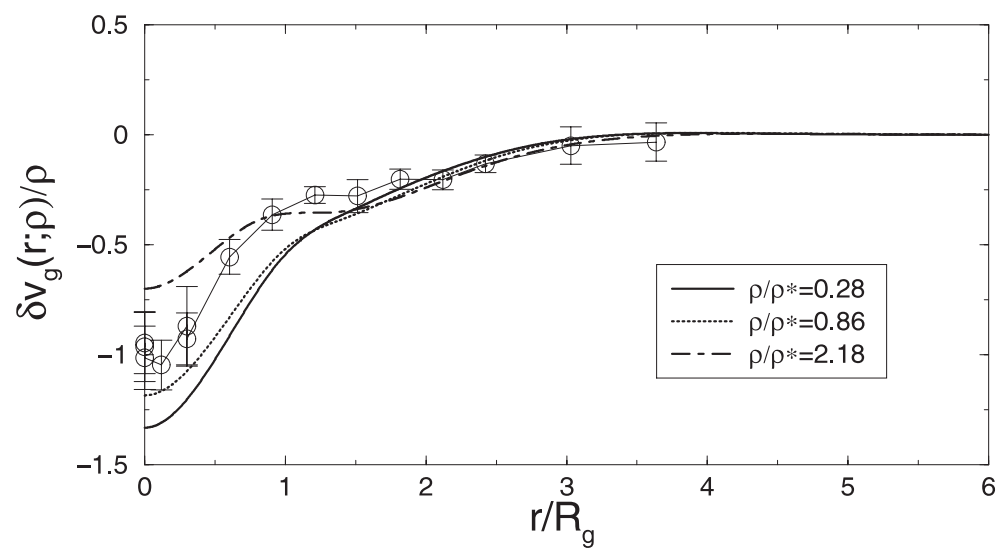

Figure 5. Explicit simulations of equation (19) show that the three-body interaction $w^{(3)}\left(\boldsymbol{r}_{i}, \boldsymbol{r}_{j}, \boldsymbol{r}_{k}\right)$ is the dominant cause of the density dependence of $v_{g}^{\text {eff }}(r ; \rho)=w^{(2)}(r)+\delta v_{\rho}(r ; \rho)$ at lower densities (taken from [32]).

These results provide a partial explanation for the thermodynamic inconsistencies found in section 2. If $v(r ; \rho)$ is equivalent to $v_{g}^{e f f}(r ; \rho)$ then only the compressibility equation has an unambiguous physical interpretation. On the other hand, one could also follow a route similar to that used to derive $v_{U}^{\text {eff }}(r ; \rho)$ to obtain a $v_{v i r}^{\text {eff }}(r ; \rho)$ which will reproduce the true pressure of an underlying many-body system through the virial equation $[24,26]^{4}$. This potential will not equal $v_{g}^{e f f}(r ; \rho)$, and so will not generate the correct pair correlations needed in equations (2) or (9). For an underlying many-body system, no single pairwise density dependent pair potential $v(r ; \rho)$ exists which can, through either the full density dependent (2) or the density independent (9) forms of the virial equation, yield the true thermodynamics.

Nevertheless, figure 6 shows that the relative overestimate found using $v_{g}^{\text {eff }}(r ; \rho)$ in the virial expression is less than the relative underestimate found when all many-body terms are ignored by only taking $w^{(2)}(r)$ into account. Using $v_{g}^{e f f}(r ; \rho)$ in the simple virial expression (11) is therefore better than either ignoring density dependence altogether (using only $w^{(2)}(r)$ ), or using the full density dependent form (2) of the virial equation. In fact for the dilute regime, $\rho / \rho^{*} \leqslant 1$, where the polymers as soft colloids coarse-graining technique is most useful, the absolute differences between the virial and compressibility routes to the EOS are quite small; similar conclusions hold for the structure generated by the two effective potentials [35]. On the other hand, for the semi-dilute regime $\left(\rho / \rho^{*}>1\right)$, the polymer EOS scales as $Z \propto \rho^{1 /(3 v-1)} \approx \rho^{1.3}$, where the Flory exponent $v \approx 0.59$ [34]. At these higher densities the RPA is excellent, which suggests that the dominant density dependence of the FT of $v_{g}^{\text {eff }}(r ; \rho)$ scales as $\hat{v}_{g}^{\text {eff }}(0 ; \rho) \sim \alpha \rho^{0.3}$, with $\alpha$ a density independent constant. This implies that $Z_{c} \sim(\alpha / 2.3) \rho^{1.3}$, while $Z_{v i r}^{0} \approx Z_{M F} \sim(\alpha / 2) \rho^{1.3}$. Using $v_{g}^{\text {eff }}(r ; \rho)$ in the virial equation at high densities results in the correct scaling exponent, but a prefactor which is about $15 \%$ too high. In contrast, ignoring the many-body terms altogether by using only $w^{(2)}(r)$ in either the virial or the compressibility equations results in $Z \sim \rho$, which scales with the wrong exponent.

4 An effective potential $v_{v i r}^{\text {eff }}(r ; \rho)$ can be defined that generates the same pressure as the full three-body virial equation when used in the standard two-body virial equation without a density dependent contribution (see e.g. [26], where it was shown explicitly that $\left.\delta v_{v i r}(r ; \rho) \neq \delta v_{U}(r ; \rho)\right)$. But since this effective potential is density dependent, one might also use the density dependent form of the virial equation, such as (2), which would generate a different effective potential. 


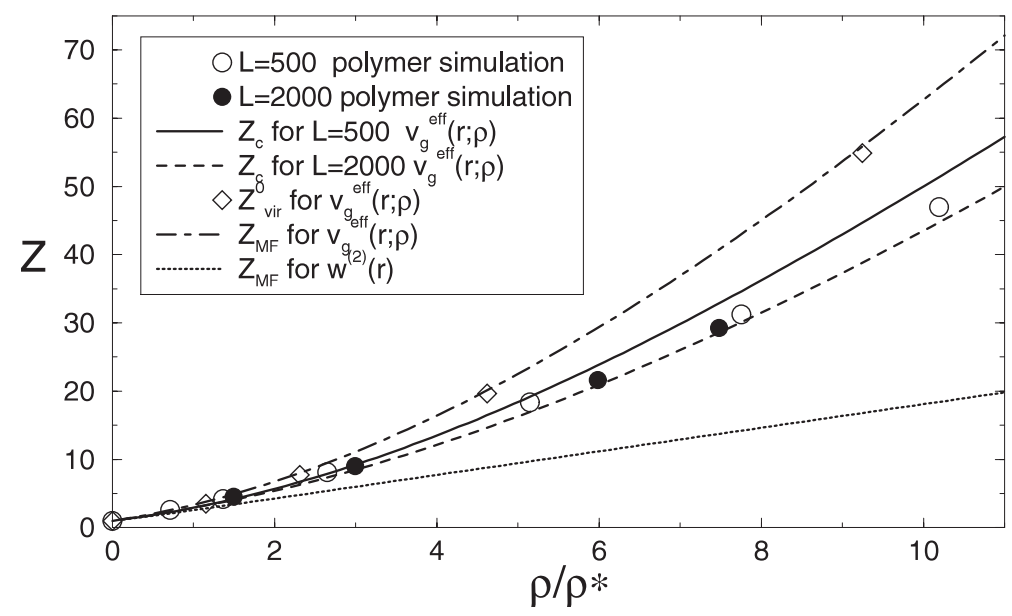

Figure 6. EOS of a polymer solution calculated in several ways: circular symbols are for direct simulations of self-avoiding walk polymers; the two $Z_{c}$ follow from equation (1); within the statistical errors of the method, they agree with the direct simulations of the full polymer system. $Z_{v i r}^{0}$ was calculated by computer simulations with $v_{g}^{\text {eff }}(r ; \rho)[18]$, and agrees well with the simpler $Z_{M F}$. In contrast to $Z_{c}$, this is not a good estimate for the true EOS. Finally, when $Z_{M F}$ is calculated without any density dependence of the pair potential, i.e. with $w^{(2)}(r)$ only, it strongly underestimates the EOS.

\subsection{Lessons for coarse-graining many-body systems}

The two previous sections have shown that there is no unique way to represent the effects of many-body interactions in effective pair interactions. This has important implications for several techniques to derive such effective pair potentials. For example, if an $a b$ initio or other higher level approach is used to generate the structure of a many-body system, then the effective pair potential that reproduces this structure will not be the same as the pair potential that correctly describes the internal energy. When the many-body interactions are weak, these differences may not be that important, but when they are strong, they may be significant. However, as seen for the polymers as soft colloids approach, using $v_{g}^{\text {eff }}(r ; \rho)$ in the virial equation may still be a better approximation than completely neglecting all manybody interactions. One might hope that similar conclusions hold for methods that derive effective pair potentials from internal energies or from structure.

In general, the stronger the many-body interactions, the more likely that extra care must be exercised when applying an effective pair potential derived by one route (i.e. structure) to extract other physical quantities (i.e. internal energy). This has implications for work done on systems with strong angular forces like water, where rather complicated effective pair potentials have be constructed to mimic certain properties [36].

A more physically motivated way to average over many-body interactions may be to derive effective pair interactions with a local density dependence, since when the local instantaneous density of a liquid is higher than the global average, one expects the relative strength of the many-body interactions to be more important there, and vice versa for lower local densities. In fact, for a number of systems, one can show explicitly that a local density dependence is equivalent to many-body interactions $[33,37]$ (the same can be done for internal degrees of freedom [38]). Although this does not imply that any many-body interaction can be consistently mapped onto a local density dependence, for those cases where it can be done a completely self-consistent thermodynamics should exist based on these effective potentials. 
Another obvious problem with a global density dependence arises when one tries to treat inhomogeneous systems. Even for argon, the LJ pair potential generates a surface tension that differs by up to $19 \%$ compared to calculations that include explicit three-body effects [41]. Again, a local density dependence would appear the more natural way to coarsegrain. Of course this opens up new problems, such as how exactly one defines the local density etc $^{5}$.

\section{Example 2. Effective one-component Hamiltonians for two-component systems}

Effective state dependent potentials naturally arise from tracing out one component in a twocomponent mixture. For uncharged systems such procedures were first carefully formulated by McMillan and Mayer in their famous theory of solutions [13]. As they, and many other authors, have stressed (see e.g. [25, 42, 43]), this works most naturally in an ensemble where the component to be traced out is treated grand-canonically. For charged systems this is no longer the case; instead, charge neutrality makes the canonical ensemble the natural choice.

As the following pedagogical examples will illustrate, whereas tracing-out procedures for uncharged systems are quite well understood, for charged systems the waters are still muddied.

\subsection{Asakura Oosawa and related models}

A popular model for polymer-colloid mixtures consists of treating the colloids as simple hard spheres (HSs) of radius $R_{c}$ and the polymers as penetrable spheres (PSs) whose interaction with the colloids is HS-like, with a cross diameter $\sigma_{c p}$ of order $R_{c}+R_{g}$, but whose interaction with the other polymers is ideal-gas-like [14]. Here I introduce a very simple and exactly solvable lattice version of this AO model, described in figure 7.

In the grand canonical ensemble the partition function for the mixture becomes

$$
\Xi_{\text {mix }}\left(\mu_{b}, \mu_{s}, N\right)=\sum_{\left\{n_{i}\right\}} \sum_{\left\{m_{i}\right\}} \exp \left(\mu_{b} \sum_{i} n_{i}+\mu_{s} \sum_{i} m_{i}\right)
$$

where $\mu_{i}$ denotes the chemical potential of each species, and the sums are over all possible configurations $\left\{n_{i}\right\}$ and $\left\{m_{i}\right\}$ of the big ('colloidal') and small ('polymeric') particles respectively. Here the number of particles at position $i$ is denoted by the occupation number $n_{i}=0,1$. Since the model is athermal, one can set $\beta=1$; in addition, the de Broglie wavelength is set to $\Lambda=1$ which simplifies notation throughout this section.

For a given configuration of the big particles $\left\{n_{i}\right\}$, there will be $M\left(\left\{n_{i}\right\}\right)$ links left for the small particles. Their partition function can then be calculated:

$$
\Xi_{\text {small }}\left(\left\{n_{i}\right\}\right)=\sum_{l=0}^{M} \frac{M^{l} z_{s}^{l}}{l !}=\exp \left[z_{s} M\left(\left\{n_{i}\right\}\right)\right]
$$

where the fugacity $z_{s}=\exp [\mu]$. For a fixed configuration $\left\{n_{i}\right\}$ of big particles, the number of free links is

$$
M\left(\left\{n_{i}\right\}\right)=2 N-4 \sum_{i} n_{i}+\sum_{\langle i j\rangle} n_{i} n_{j}
$$

since there are two links for each site, each big particle excludes four links, but when two big particles touch they only exclude seven links, since one is doubly excluded, as illustrated

5 An interesting recent example for an $N V T$ simulation can be found in [39]. However, the original effective potential studied was of the $v_{U}^{\text {eff }}(r ; \rho)$ form [40], while in [39] it is used in a virial equation like equation (2). The lack of thermodynamic self-consistency found by these authors stems in part from their use of a density dependent interaction without a careful analysis of its origin. 


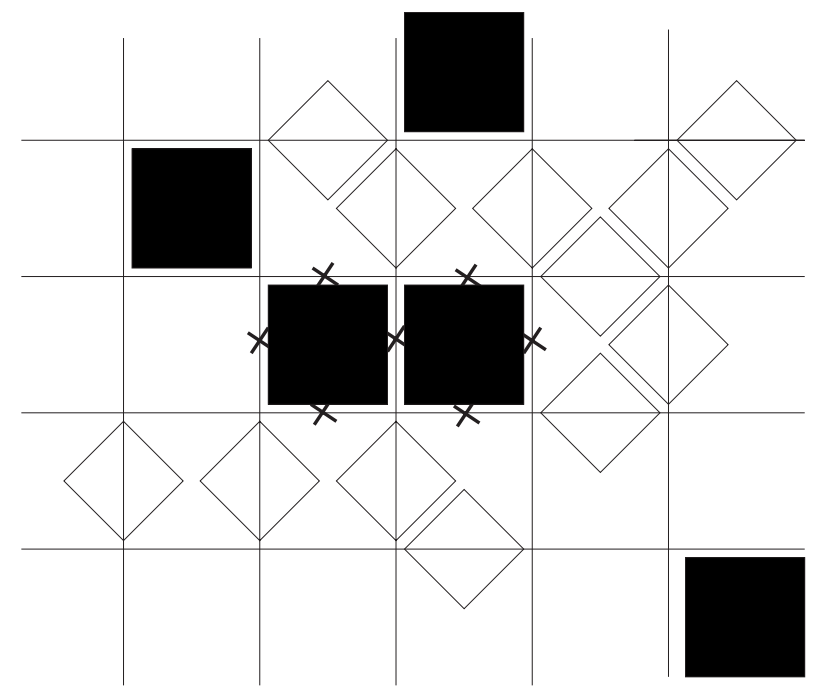

Figure 7. A two-dimensional lattice version of the AO model. The big squares live on the $N$ sites, and the small ones live on the $2 N$ links. The big squares are hard, and cannot overlap each other, and similarly the big and small squares exclude each other. The small squares are ideal w.r.t. each other, and so multiple occupancy of links not excluded by big particles is possible. The crosses on the central big squares show which links they exclude from the small particles.

by the crosses in figure 7 . This increase in the effective volume available (and concomitant entropy) for the small particles when two big ones touch is the origin of the osmotic depletion effect [14]. A large enough fugacity of the small particles can induce an entropy driven phase separation of the big particles.

With the explicit form (25) for the available 'free volume' $M\left(\left\{n_{i}\right\}\right)$, the full partition function (23) simplifies to

$$
\Xi_{m i x}\left(\mu_{b}, \mu_{s}, N\right)=\exp \left[2 z_{s} N\right] \sum_{\left\{n_{i}\right\}} \exp \left[\left(\mu_{b}-4 z_{s}\right) \sum_{i} n_{i}+z_{s} \sum_{\langle i j\rangle} n_{i} n_{j}\right]
$$

This illustrates the essence of the McMillan-Mayer tracing-out procedure: the sums over states in the partition function of the original two-component system are rewritten as a single sum over the states of an effective one-component system. There is no need to invoke any BornOppenheimer type timescale separation arguments; the procedure might even be viewed as simply a mathematical trick. One could just as well trace out the big particles. The particular advantage of tracing out the small particles is that the effective one-component system is simpler to treat, since it can be mapped onto a binary lattice gas model with an effective chemical potential $\mu_{b}^{e f f}=\mu_{b}-4 z_{s}$ and an effective Hamiltonian that, in turn, is decomposable as a pairwise sum over nearest neighbours with an interaction strength $\epsilon^{\text {eff }}=-z_{s}$. By mapping onto an Ising spin system in two dimensions, this model, with its entropically driven phaseseparation transition, can be exactly solved, as shown previously for a closely related model of non-additive hard squares [44]. Mathematically these models are a special cases of the more general decorated Ising model introduced by Widom [45].

Keeping both species grand-canonical facilitates the mapping to an Ising model, but it is just as easy to integrate out the small particles in a semi-grand ensemble, i.e. fixing $\left(N_{b}, z_{s}, N\right)$, 
resulting in an effective partition function of the form

$$
Z_{\text {mix }}\left(N_{b}, z_{s}, N\right)=\exp \left[z_{s}\left(2 N-4 N_{b}\right)\right] \sum_{\left\{n_{i}\right\}^{\prime}} \exp \left[z_{s} \sum_{\langle i j\rangle} n_{i} n_{j}\right]
$$

where $\left\{n_{i}\right\}^{\prime}$ denotes all possible ways of arranging $N_{b}$ particles on a lattice of $N$ sites. Again, as long as $z_{s}$ is fixed, $Z_{\text {mix }}$ can be interpreted as an effective one-component system, interacting with an effective Hamiltonian of the form

$$
\beta H^{e f f}\left(N_{b}, z_{s}, N ;\left\{n_{i}\right\}\right)=H_{b b}-z_{s} 2 N+z_{s} 4 N_{b}-z_{s} \sum_{\langle i j\rangle} n_{i} n_{j}
$$

with a configuration independent 'volume term', and a pairwise decomposable pair interaction. $H_{b b}$ is the bare hard-core big-big interaction that enforces the single occupancy constraint. All the standard statistical mechanics for such one-component systems can now be brought to bear to calculate correlations and phase behaviour. The volume term contributes to thermodynamic quantities, but not to the phase behaviour [9, 43].

The tracing-out procedure can also be done in the canonical ensemble, keeping both $N_{b}$ and $N_{s}$ fixed:

$Z_{\text {mix }}\left(N_{b}, N_{s}, N\right)=\sum_{\left\{n_{i}\right\}^{\prime}}\left(M\left(\left\{n_{i}\right\}\right)\right)^{N_{s}}=\sum_{\left\{n_{i}\right\}^{\prime}} \exp \left[N_{s} \log \left[2 N-4 N_{b}+\sum_{\langle i j\rangle} n_{i} n_{j}\right]\right]$.

But now the effective one-component system is no longer equivalent to a system with a pairdecomposable effective Hamiltonian; at best it can be rewritten as

$H^{e f f}\left(N_{b}, N_{s}, N ;\left\{n_{i}\right\}\right)=H_{b b}-N_{s} \log \left[2 N-4 N_{b}\right]-N_{s} \log \left[1+\frac{1}{2 N-4 N_{b}} \sum_{i j} n_{i} n_{j}\right]$.

In the limit of only two big particles the logarithms can be expanded and the pair term in the Hamiltonian reduces to the same form as found (in the same limit) for the grand-canonical tracing-out procedure. But for a larger number of big particles this ceases to be true: the Hamiltonian can no longer be written as a sum over independent many-body interactions of the form of equations (13) or (21). The effect on $H^{\text {eff }}\left(N_{b}, N_{s}, N ;\left\{n_{i}\right\}\right)$ of changing the number of pairs in a configuration $\left\{n_{i}\right\}$ by one depends on the configuration of all other pairs in the system. The McMillan-Mayer mathematical tracing-out procedure does not lead to such a useful simplification in the canonical ensemble as it does in the semi-grand ensemble.

Similar manipulations can be performed for off-lattice two-component systems such as the original AO model [14]. Integrating out the $N_{p}$ PS polymeric particles for a fixed configuration $\left\{\boldsymbol{r}_{i}\right\}$ of $N_{c}$ colloidal HSs results in effective Hamiltonians of the form

$$
\begin{aligned}
& H^{e f f}\left(N_{c}, z_{p}, V ;\left\{\boldsymbol{r}_{i}\right\}\right)=H_{c c}+\Omega\left(N_{c}, z_{p}, V ;\left\{\boldsymbol{r}_{i}\right\}\right) \\
& H^{\text {eff }}\left(N_{c}, N_{p}, V ;\left\{\boldsymbol{r}_{i}\right\}\right)=H_{c c}+F\left(N_{c}, N_{p}, V ;\left\{\boldsymbol{r}_{i}\right\}\right)
\end{aligned}
$$

for the semi grand and canonical ensembles respectively. $H_{c c}$ is the bare colloid Hamiltonian. $\Omega\left(N_{c}, z_{p}, V ;\left\{\boldsymbol{r}_{i}\right\}\right)$ is the grand potential and $F\left(N_{c}, N_{p}, V ;\left\{\boldsymbol{r}_{i}\right\}\right)$ is the Helmholtz free energy of an inhomogeneous system of PS particles in the external field of the colloids. In fact, the free energy form of these effective Hamiltonians holds for a much wider class of two-component systems $[9,43]$. Integrating over all possible configurations $\left\{\boldsymbol{r}_{i}\right\}$ of the colloidal particles leads to the full two-component partition function. For the AO model these Hamiltonians take a particularly simple form since

$$
\Omega\left(N_{c}, z_{p}, V ;\left\{\boldsymbol{r}_{i}\right\}\right)=-z_{p} V^{\text {free }}\left(N_{c}, V ;\left\{\boldsymbol{r}_{i}\right\}\right)
$$


is the grand potential of an ideal gas in the accessible free volume $V^{\text {free }}\left(N_{c}, V ;\left\{\boldsymbol{r}_{i}\right\}\right)$ and similarly

$$
F\left(N_{c}, N_{p}, V ;\left\{\boldsymbol{r}_{i}\right\}\right)=N_{p} \log \left[N_{p}\right]-N_{p}-N_{p} \log \left[V^{\text {free }}\left(N_{c}, V ;\left\{\boldsymbol{r}_{i}\right\}\right)\right]
$$

is the Helmholtz free energy of an ideal gas of $N_{p}$ particles in the accessible free volume.

The calculation of $V^{\text {free }}\left(N_{c}, V ;\left\{\boldsymbol{r}_{i}\right\}\right)$, the direct analogue of $M\left(\left\{n_{i}\right\}\right)$ in the AO lattice models, simplifies dramatically if the size ratio $R_{g} / R_{c} \leqslant 0.1547$. There are then no triplet or higher order overlaps of the exclusion zones, and the accessible free volume can be written as

$$
V^{\text {free }}\left(N_{c}, V ;\left\{\boldsymbol{r}_{i}\right\}\right)=V-N_{c} V_{1}+\sum_{i<j} V_{2}\left(r_{i j}\right)
$$

where $V_{1}=\frac{4}{3} \pi\left(\sigma_{c p}\right)^{3}$ is the volume excluded by each colloidal particle, and $V_{2}(r)$ has the standard AO form [14], depending only on the relative separation $r$ of two particles. (Note the correspondence with equation (25) for the lattice model, where $V=2 \mathrm{~N}, V_{1}=4$ and the sum over $V_{2}(r)$ is replaced by a nearest neighbour lattice sum.) The effective Hamiltonians of equations (31) and (32) simplify to

$$
\begin{gathered}
H^{e f f}\left(N_{c}, z_{p}, V ;\left\{\boldsymbol{r}_{i}\right\}\right)=H_{c c}-z_{p} V+z_{p} N_{c} V_{1}-z_{p} \sum_{i<j} V_{2}(r) \\
H^{e f f}\left(N_{c}, N_{p}, V ;\left\{\boldsymbol{r}_{i}\right\}\right)=H_{c c}+N_{p} \log \left[\frac{N_{p}}{V-N_{c} V_{1}}\right]-N_{p} \\
-N_{p} \log \left[1+\frac{1}{V-N_{c} V_{1}} \sum_{i<j} z_{p} V_{2}(r)\right] .
\end{gathered}
$$

Just as was found for the lattice model, the canonical tracing-out procedure leads to a Hamiltonian that cannot be written as a sum over independent interactions of the form of equations (13) or $(21)^{6}$. In contrast, the semi-grand $H^{\text {eff }}\left(N_{c}, z_{p}, V,\left\{\boldsymbol{r}_{i}\right\}\right)$ takes on the more useful form of a 'volume term' plus a sum over an effective pair potential. The interpretation of the latter will now be treated in more detail.

While volume terms in equation (36) are important to make contact with the full twocomponent system, as worked out very clearly and in great detail by Dijkstra et al [46], they do not contribute to the phase behaviour of the system. For $R_{g} / R_{c}<0.1547$ the simplified pairwise Hamiltonian

$$
H^{e f f}\left(N_{c}, z_{p}, V\right)=H_{c c}+\sum_{i<j} v_{z}^{e f f}\left(r ; z_{p}\right)
$$

with $v_{z}^{e f f}\left(r ; z_{p}\right)=-z_{p} V_{2}(r)$, completely determines the phase behaviour and correlations of the colloidal system. An entirely self-consistent one-component osmotic thermodynamics can be defined based on the Hamiltonian (38). This means, for example, that the compressibility equation (1) generates the same osmotic EOS as the virial equation:

$$
Z_{v i r}=\frac{\Pi\left(N_{c}, z_{p}, V\right)}{\rho_{c}}=1-\frac{2 \pi}{3} \rho_{c} \int_{0}^{\infty} r^{3} \frac{\partial v_{z}^{e f f}\left(r ; z_{p}\right)}{\partial r} g_{c c}(r) \mathrm{d} r
$$

where $\Pi\left(N_{c}, z_{p}, V\right)$ is the osmotic pressure ${ }^{7}$. Since $z_{p}$ is a fixed external parameter, $v_{z}^{e f f}\left(r ; z_{p}\right)$ is in fact not state dependent within this one-component picture. Thus, the McMillan-Mayer tracing-out procedure results in a system that can be exactly mapped onto a

6 The small differences between the volume terms in the lattice and off-lattice versions of the AO model stem from kinetic energy terms that are ignored in the former case.

7 Note that adding the explicit volume term $\left(-\partial F_{0} / \partial V\right)$ to the compressibility and virial equations would generate the thermodynamics of the full two-component system. 
classical one-component fluid with a state independent pair potential ${ }^{8}$. One must simply keep in mind that the resultant thermodynamics quantities are osmotic-defined w.r.t. a reservoir containing PS particles only. Similarly, since the McMillan-Mayer procedure is essentially a mathematical trick to facilitate calculating the sums in the partition function, very little information can a priori be extracted about the effective dynamics of the one-component system. For that, further physically motivated arguments and approximations are needed.

Now suppose one were working in the canonical ensemble, and naively used the AO depletion potential $v_{z}^{e f f}\left(r ; z_{p}\right)$, as has sometimes been done in the literature. Then $z_{p}=$ $z_{p}\left(\rho_{c}, \rho_{p}\right)$ would no longer be an external parameter but would instead depend on the state of the system. For example, for small size ratios a good approximation is $z_{p} \approx \rho_{p} /\left(1-\rho_{c} V_{1}\right)[43,46]$. One might be tempted to define an effective one-component thermodynamics by fixing $\rho_{p}$, and treating it as an external parameter, as might be natural in an experiment. The potential then takes on the $\rho_{c}$ dependent form $v_{\rho}^{e f f}\left(r ; \rho_{c} ; \rho_{p}\right)$. If this potential is taken as given, without enquiring as to its origins, as was done, for example in section 2 , then the compressibility and virial equations take forms similar to equations (1) and (2) respectively, since $v_{\rho}^{e f f}$ depends on $\rho_{c}$. One might expect that the ensuing thermodynamics would again be that of the osmotic system, but now neither the derived virial nor the derived compressibility equation is correct. For the present case of a fixed $\rho_{p}$, the compressibility equation (1) using $v_{\rho}\left(r ; \rho_{p}, \rho_{c}\right)$ does not result in the correct osmotic pressure because $z_{p}$ varies when $\rho_{c}$ is changed. On the other hand, using $v_{\rho}^{e f f}\left(r ; \rho_{c}, \rho_{p}\right)$ in the simpler virial equation (39), i.e. one without a density derivative, does generate the correct osmotic EOS, as follows from the following arguments: the thermodynamic properties are independent of ensemble, so, for a given state point $\left(N_{c}, N_{p}, V\right)$, the osmotic pressure is the same as in the semi-grand ensemble at a state point $\left(N_{c}, z_{p}, N\right)$ such that $\left\langle N_{p}\right\rangle_{z p, N c, V}=N_{p}$. There the potential $v_{\rho}^{\text {eff }}\left(r ; \rho_{p}, \rho_{c}\right)=v_{z}^{\text {eff }}\left(r ; z_{p}\right)$ generates the correct pair correlations $g_{c c}(r)$, and also the correct virial pressure $\Pi\left(N_{c}, N_{p}, V\right)$ through equation (39). But the apparent relevance of this osmotic virial equation within the canonical ensemble is deceptive-it only follows because it can be derived in the semi-grand ensemble, and used at a state point where $v_{\rho}^{\text {eff }}\left(r ; \rho_{c}, \rho_{p}\right)=v_{z}^{\text {eff }}\left(r ; z_{p}\right)$.

The lack of consistency between virial and compressibility routes should, of course, not be surprising, since a careful tracing-out procedure demonstrates that the effective Hamiltonian (37) for the canonical ensemble cannot be decomposed into a sum over independent pair potentials. Therefore, using the AO pair potential in this ensemble is not rigorously justified, except for instances where parallels with the semi-grand ensemble can be made ${ }^{9}$.

In summary then: for a fixed $z_{p}$, a completely self-consistent one-component thermodynamics can be derived in the semi-grand ensemble for an AO system. But within the canonical ensemble, a McMillan-Mayer style tracing-out procedure leads to an effective Hamiltonian that cannot easily be written as a sum over pair and higher order interaction terms; the ensuing one-component system does not have a simple interpretation as an effective liquid. Moreover, if the AO depletion potential is naively applied in the canonical ensemble for fixed $\rho_{p}$, then the density dependence of the pair potential again leads to an apparent lack of consistency between different routes to osmotic thermodynamics. In contrast to the case

8 For larger size ratios, the mapping results in three-body and higher order interactions, leading to similar problems to those discussed in section 3 when one tries to derive an effective pair potential. Including interactions between the small particles will also lead to effective three-body and higher order terms.

9 Note, however, that in the limit $N_{c}=2$, both the canonical and the grand-canonical ensembles do result in the same pair potential, as can be seen by expanding the log in equation (37). A similar conclusion holds for higher order interactions, should they be relevant. However, great care must be applied when these low density effective interactions are used at finite colloid densities in the canonical ensemble. 
studied in section 3 , where the effective density dependent potential $v_{g}^{\text {eff }}(r ; \rho)$ that arises from tracing out three-body interactions generates the correct thermodynamics only through the compressibility equation, here only the virial equation (39) should be used.

\subsection{Debye-Hückel model}

The effective interactions and resulting phase behaviour of charge-stabilized colloidal suspensions have been the subject of much recent debate [8, 47]. In contrast to uncharged mixtures, global charge neutrality implies that the canonical ensemble is the natural choice in which to integrate out the co- and counterions to derive an effective one-component colloidal picture. And this explains in part why the problem is so difficult, since, as was shown in the previous subsection, tracing out one component in the canonical ensemble does not necessarily lead to an obvious description in terms of independent (many-body) interactions. In addition, direct computer simulations of the full mixture are greatly complicated by the long-range nature of the Coulomb interactions and the large length scale differences between a typical colloidal particle and the co- and counterions.

Rather than attempting yet another tracing-out procedure, this section has a much more modest goal, namely to illustrate pitfalls that arise from a naive application of a very simple textbook density dependent potential of the Debye-Hückel screened Yukawa form

$$
\beta v_{D H}(r ; \rho)=\frac{Z^{2}}{r} \exp [-\kappa(\rho) r] .
$$

Here $Z$ is the charge of the colloidal particle, and $\kappa(\rho)=\sqrt{4 \pi Z \rho}$ is the screening parameter in the absence of salt. The Bjerrum length $\lambda_{B}=\beta e^{2} / \epsilon$, with $e$ the elemental charge and $\epsilon$ the dielectric constant, has been set to 1 , to simplify the notation. Since $\kappa$ depends on the overall density (through charge neutrality), it should come as no surprise that a simple application of the compressibility equation (1) and the virial equation (2) do not generate the same thermodynamics. Since equation (40) is an integrable potential,

$$
\beta \hat{v}(k=0 ; \rho)=\frac{4 \pi Z^{2}}{\kappa^{2}}=\frac{Z}{\rho},
$$

its thermodynamic behaviour resembles that of a mean-field fluid [10] for large $\rho$ or (very) small effective $Z$, where the RPA closure should be quite accurate. Thus the two routes lead to

$$
Z_{c}^{R P A}=1+Z
$$

which can be interpreted as the ideal EOS of the colloids and $Z$ counterions, and

$Z_{v i r}=\left(1+\frac{1}{2} Z\right)-\frac{2}{3} \beta \pi \rho \int r^{2}\left[h(r)\left(r \frac{\partial v_{D H}(r)}{\partial r}\right)-g(r) 3 \rho \frac{\partial v_{D H}(r)}{\partial \rho}\right]$.

Even the leading term in the virial equation differs from the compressibility equation. Since both the $r$ and $\rho$ derivatives of $v_{D H}(r ; \rho)$ are always negative, the second two terms of $Z_{v i r}$ both reduce its value w.r.t. the leading $Z_{M F}=\left(1+\frac{1}{2} Z\right)$ term ${ }^{10}$, increasing the difference between the two routes even further. The present discrepancy originates not in the lack of consistency of the closure, but rather in the naive application of a density dependent pair potential. A more careful analysis of the underlying two-component colloid + counterion system shows that volume terms must also be taken into account [47], but these do not bring the two routes

${ }^{10}$ At least in the regime where the RPA is a reasonable approximation. Note that the virial equation can be exactly solved in the RPA approximation [22] 
any closer together. The only way to know which (if any) of the two routes is the more reliable would be to derive them from a careful analysis of the full tracing-out procedure ${ }^{11}$.

The arguments above may have repercussions for the modelling of liquid metals by effective density dependent pair potentials. There, the Ascarelli-Harrison form of the virial equation (2) has often been applied, but, as other examples in this paper show, it is not yet clear whether this equation is reliable. In fact, a recent careful derivation of an effective onecomponent virial equation within a two-component electron ion picture by Chihara et al [48] results in a form without an explicit density derivative.

Finally, it appears that effective potentials in charged systems will always be density dependent due to charge neutrality. But this same charge neutrality implies that a twocomponent system can be viewed effectively as a one-component one. For example, all the partial structure factors have the same $k \rightarrow 0$ limit, up to trivial prefactors, i.e. they are not independent. The density dependence in charged systems most likely has a different character from that found in uncharged systems.

\section{Conclusions}

The overall conclusion of this paper can be summarized by the statement

an effective density dependent pair potential $\boldsymbol{v}(\boldsymbol{r} ; \boldsymbol{\rho})$ cannot be properly interpreted without reference to the coarse-graining procedure by which it was derived.

This was illustrated by a number of explicit examples. Although these were mainly drawn from the field of soft matter, the principles should be equally valid for the effective pair potentials used in other branches of condensed matter science. The only examples where an effective pair potential could be rigorously interpreted as part of an effective Hamiltonian were the AO model for $R_{g} / R_{c} \leqslant 0.1547$ and its lattice analogue. Other potentials like $v_{g}^{\text {eff }}(r ; \rho)$, which reproduces the correct underlying pair structure, cannot be formally interpreted as part of an effective Hamiltonian. Instead, they should be viewed as mathematical devices to calculate desired properties from a particular equation [23-26].

Some of the more detailed conclusions are listed below.

- A pair potential that depends on global density, $v(r ; \rho)$, does not generally lead to the same thermodynamics in the canonical and grand-canonical ensembles.

- The only effective potential which leads to the correct thermodynamics through the compressibility equation (1) is $v_{g}^{\text {eff }}(r ; \rho)$

- The status of the Ascarelli-Harrison [16] form of the virial equation (2), with an added $\partial v(r ; \rho) \partial \rho$ term, is suspect. For uncharged systems and many-body systems, counter-examples can easily be constructed, where it does not apply and even makes thermodynamic inconsistencies worse. Whether it is valid for liquid metals or other charged systems remains to be proved.

- There is no unique way to represent the effect of many-body interactions as density dependent pair interactions [23-26]. The potential that correctly reproduces the structure, $v_{g}^{e f f}(r ; \rho)$, will not generate the right internal energy when used in the energy equation etc. At best, one can pick a $v^{e f f}(r ; \rho)$ that performs well for the particular physical properties one is interested in. For example, the parameters commonly used in the LJ potential to model liquid argon should be viewed as a compromise between those that correctly reproduce the internal energy, the virial pressure and the structure [26].

${ }^{11}$ One example where equation (2) appears to be better than forms without the density derivative can be inferred from the discussion of the Debye-Hückel model by Belloni [8]. By adding the explicit volume term to his application of the Debye-Hückel potential in equation (2), the virial pressure closely resembles an approximate form derived from a two-component picture. 
- The previous statement implies a link between problems with the transferability of an effective pair potential, and problems with its representability. For example, if manybody interactions generate a large relative density dependence in $v^{e f f}(r ; \rho)$, then the potential derived by a given route at $\rho_{1}$ will differ significantly from the one derived at $\rho_{2}$, leading to transferability problems. This large density dependence also implies important differences between say $v_{U}^{e f f}(r ; \rho)$ and $v_{g}^{e f f}(r ; \rho)$, leading to representability problems.

- A McMillan-Mayer type tracing-out procedure for a two-component system in the canonical ensemble does not lead to an effective one-component Hamiltonian that can be written as a sum of independent many-body interactions.

- The Debye-Hückel potential, like all such density dependent pair potentials, leads to thermodynamic inconsistencies when it is naively used in an effective one-component picture.

- Obvious problems arise when potentials like $v(r ; \rho)$ are used in inhomogeneous systems. There it would make more sense to coarse-grain to potentials that depend on a measure of the local density. In some cases, this can be shown to be equivalent to a many-body interaction approach.

I finish with the question: when do these results matter? The picture may not be as bleak as it was painted in the discussions above. Indeed, in the case of argon, the differences between $v_{g}^{\text {eff }}(r ; \rho)$ and $v_{U}^{\text {eff }}(r ; \rho)$ are small, so that a single compromise effective pair potentials works admirably well for the liquid phase. Many examples can be found for softmatter systems where ignoring the many-body forces altogether-simply using $w^{(2)}(r)$ in the expansion of an $H^{e f f}$ — works quite well. Examples include systems described by short-range depletion potentials [43, 49, 50], or effective interactions derived for star-polymers [51] or dendrimers [52]. Moreover, in other cases, such as the polymers as soft colloids example of section 3 , using $v_{g}^{\text {eff }}(r ; \rho)$ in the simple virial equation may still be a better approximation than ignoring many-body effects altogether. Similar conclusions should hold for other condensed matter systems, but more work is needed to determine when the problems of representability and density dependence become important for the description of such materials. Nevertheless, in each case the particular coarse-graining procedure used to derive $v^{\text {eff }}(r ; \rho)$ must be kept in mind - the naive consumer of effective pair potentials should beware.

\section{Acknowledgments}

I acknowledge support from the Isaac Newton Trust, Cambridge, and thank Neil Ashcroft, Peter Bolhuis, Bob Evans, Daan Frenkel, Martin van der Hoef, Gerhard Kahl, Hartmut Löwen, Paul Madden, Richard Sear, Vincent Krakoviack and Patrick Warren for many useful discussions. But most of all, I would like to thank Jean-Pierre Hansen, who has been closely involved in this work, and who has been a great inspiration to me, both as a scientist and as a person. It is with great pleasure that I dedicate this paper to the honour of his 60th birthday.

\section{References}

[1] Axilrod B M and Teller E 1943 J. Chem. Phys. 11299

[2] Ashcroft N W and Stroud D 1978 Solid State Phys. 331

[3] Daw M S and Baskes M I 1984 Phys. Rev. B 296443

[4] Foiles S M 1985 Phys. Rev. B 323409

[5] Jacobsen K W, Norskov J K N and Puska M J 1987 Phys. Rev. B 357423

[6] Finnis M W and Sinclair J E 1984 Phil. Mag. A 5045

[7] Ercolessi F, Tosatti E and Parrinello M 1986 Phys. Rev. Lett. 57719

[8] Belloni L 2000 J. Phys.: Condens. Matter 12 R549

[9] Likos C N 2001 Phys. Rep. 348267 
[10] Louis A A 2001 Phil. Proc. R. Soc. A 359939

[11] Reatto L 1986 Phil. Mag. A 5837

Reatto L, Levesque D and Weis J J 1986 Phys. Rev. A 333451

[12] See e.g.

Robertus C, Philipse W H, Joosten J G H and Levine Y K 1989 J. Chem. Phys. 904482

Cametti P, Codastefano P, Tartaglia P, Rouch J and Chen S H 1990 Phys. Rev. Lett. 641461

Bouaskarne M, Amokrane S and Regnaut C 1999 J. Chem. Phys. 1112151

[13] McMillan W G and Mayer J E 1945 J. Chem. Phys. 13276

[14] Asakura S and Oosawa F 1958 J. Polym. Sci. Polym. Symp. 33183 Vrij A 1976 Pure Appl. Chem. 48471

[15] Hansen J P and McDonald I R 1986 Theory of Simple Liquids 2nd edn (London: Academic)

[16] Ascarelli P and Harrison R 1969 Phys. Rev. Lett. 22285

[17] Louis A A, Bolhuis P G, Hansen J-P and Meijer E J 2000 Phys. Rev. Lett. 852522

[18] Bolhuis P G, Louis A A, Hansen J-P and Meijer E J 2001 J. Chem. Phys. 1144296

[19] Louis A A, Bolhuis P G and Hansen J-P 2000 Phys. Rev. E 627961

[20] Lang A, Likos C N, Watzlawek M and Löwen H 2000 J. Phys.: Condens. Matter 125087

[21] Likos C N, Lang A, Watzlawek M and Löwen H 2001 Phys. Rev. E 63031206

[22] Louis A A 2002 unpublished

[23] Barker J A, Henderson D and Smith W R 1969 Mol. Phys. 17579

[24] Casanova G, Dulla R J, Jonah D A, Rowlinson J S and Saville G 1970 Mol. Phys. 18589

[25] Rowlinson J S 1984 Mol. Phys. 52567

[26] van der Hoef M A and Madden P A 1999 J. Chem. Phys. 1111520

[27] Kirkwood J G 1952 J. Chem. Phys. 20929

[28] Henderson R L 1974 Phys. Lett. A 49197 Chayes J T and Chayes L 1984 J. Stat. Phys. 36471

[29] Evans R 1990 Mol. Simul. 4409

[30] Reatto L and Tau M 1987 J. Chem. Phys. 866474

[31] Attard P 1991 Phys. Rev. A 453659

[32] Bolhuis P G, Louis A A and Hansen J-P 2001 Phys. Rev. E 64021801

[33] Carlsson A E 1990 Solid State Phys. 431

[34] de Gennes P G 1979 Scaling Concepts in Polymer Physics (Ithaca, NY: Cornell University Press)

[35] Bolhuis P G and Louis A A 2002 Macromolecules 351860

[36] See e.g.

Malescio G, Franzese G, Pellicane G, Skibinsky A, Buldyrev S V and Stanley H E 2002 J. Phys.: Condens. Matter 142193 and references therein

[37] Pagonabarraga I and Frenkel D 2000 Mol. Simul. 25167

[38] Sear R P 2000 Phys. Rev. E 61651

[39] Almarza N G, Lomba E, Ruiz G and Tejero C F 2001 Phys. Rev. Lett. 862038

[40] Tejero C F and Baus M 1998 Phys. Rev. E 574821

[41] Barker J A 1993 Mol. Phys. 80815

[42] Lekkerkerker H N W, Poon W C K, Pusey P N, Stroobants A and Warren P B 1992 Europhys. Lett. 20559

[43] Dijkstra M, van Roij R and Evans R 1999 Phys. Rev. E 595744

[44] Frenkel D and Louis A A 1992 Phys. Rev. Lett. 683363

[45] Widom B 1967 J. Chem. Phys. 463324

[46] Dijkstra M, van Roij R and Evans R 2000 J. Chem. Phys. 1134799

[47] See e.g.

van Roij R and Hansen J P 1997 Phys. Rev. Lett. 793082

Graf H and Löwen L 1998 Phys. Rev. E 575744

van Roij R, Dijkstra M and Hansen J P 1999 Phys. Rev. E 592010

Warren P B J. Chem. Phys. 1124683

Chan D Y C 2001 Phys. Rev. E 63061806

[48] Chihara J, Fukumoto I, Yamagiwa M and Totsuji H 2001 J. Phys.: Condens. Matter 137183

[49] Meijer E J and Frenkel D 1994 J. Chem. Phys. 1006873

[50] Dijkstra M, Brader J M and Evans R 1999 J. Phys.: Condens. Matter 1110079

[51] Likos C N, Löwen H, Watzlawek M, Abbas B, Jucknischke O, Allgaier J and Richter D 1998 Phys. Rev. Lett. 804450

Watzlawek M, Likos C N and Löwen H 1999 Phys. Rev. Lett. 825289

[52] Likos C N, Schmidt M, Löwen H, Ballauff M, Pötschke D and Lindner P 2001 Macromolecules 342914 